\title{
Análisis de la inclusión económica de los jóvenes al Mercado laboral en ecuador en el periodo 2009 al 2019
}

\section{Analysis of the economic inclusion of young people Labor market in ecuador in the period 2009 to 2019}

DOI: $10.46932 / \mathrm{sfjdv2n4-083}$

Received in: March 1st, 2021

Accepted in: May 30th, 2021

\author{
Melina Veliz Andrade \\ Estudiante carrera de economía agropecuaria \\ Universidad Técnica de Machala. Km 5 1/2 vía Pasaje \\ Machala-Ecuador \\ E-mail: mveliz1@utmachala.edu.ec \\ Andrea Vega Granda \\ Universidad Técnica de Machala. Km 5 1/2 vía Pasaje \\ Machala-Ecuador \\ Economista \\ E-mail: avega@utmachala.edu.ec \\ Víctor Garzón Montealegre \\ Universidad Técnica de Machala. Km 5 1/2 vía Pasaje \\ Machala-Ecuador \\ Economista \\ E-mail:vgazon@utmachala.edu.ec \\ Jessica Quezada Campoverde \\ Universidad Técnica de Machala. Km 5 1/2 vía Pasaje \\ Machala-Ecuador \\ Ingeniera agrónoma \\ E-mail: jquezada@utmachala.edu.ec

\section{Eveligh Prado-Carpio} \\ Universidad Técnica de Machala. Km 5 1/2 vía Pasaje \\ Machala-Ecuador \\ Dra. En Ciencias Agrarias. PhD \\ E-mail: eprado@utmachala.edu.ec
}

\section{RESUMEN}

El presente artículo tiene como objetivo analizar la inclusión económica juvenil en el mercado laboral del Ecuador en el periodo 2009 al 2019, tomando como referencia información de fuentes secundarias, correspondientes a la recopilación de evidencias investigativas como la Organización Internacional del Trabajo (OIT), la Comisión Económica para América Latina y el Caribe (CEPAL), el Ministerio de Trabajo, el Instituto Nacional de Estadística y Censos (INEC), entre otras revisiones bibliográficas enfocadas en la situación real que atraviesan la mayoría de los jóvenes en el país, en base a los resultados obtenidos se establecen los factores más relevantes que determinan la empleabilidad, como son la educación, la oferta y demanda laboral, las condiciones socio-económicas, instituciones labores, entre 
otros que como consecuencia, ha disminuido el pleno empleo, el subempleo y por lo contrario el desempleo ha aumentado.

Palabras clave: Inclusión económica, mercado laboral, inserción juvenil.

\section{ABSTRACT}

This article aims to analyze youth economic inclusion in the Ecuadorian labor market in the period 2009 to 2019, taking as reference information from secondary sources, corresponding to the compilation of investigative evidence such as the International Labor Organization (ILO), the Economic Commission for Latin America and the Caribbean (ECLAC), the Ministry of Labor, the National Institute of Statistics and Censuses (INEC), among other bibliographic reviews focused on the real situation that the majority of young people in the country go through, based on the results obtained establish the most relevant factors that determine employability, such as education, labor supply and demand, socio-economic conditions, labor institutions, among others, which as a consequence, has decreased full employment, underemployment and on the contrary, unemployment has increased.

Keywords: Economic inclusion, labor market, youth insertion.

\section{INTRODUCCIÓN}

Como un paso a la adultez y enfrentando los primeros obstáculos de la emancipación, el trabajo para un joven es la necesaria transición para formar parte de la sociedad y del mundo que le rodea, formando su identidad como individuo llevándolo a tomar decisiones en los cuales ya sea el entorno en el que está o sus capacidades darán la clara posibilidad de éxito o fracaso en este desarrollo. (CEPAL, 1998).

Se ha hecho énfasis en este dicho de que "la juventud es el futuro del país" cuando en la actualidad, la juventud es una generación en desventaja, principalmente por la falta de empleo no solo para los jóvenes sino para todos, además otra desventaja es la poca inversión en un personal joven por parte de las empresas y el Estado, es claro que anteriormente se han estructurado formas para potenciar el empleo de los jóvenes, sin embargo, no se las ha ejecutado como se las había planteado.

Por lo tanto, por medio de este trabajo investigativo se tiene como objetivo analizar la inclusión económica con la juventud ecuatoriana en el mercado laboral en el periodo del 2009 al 2019.

Según la Organización Internacional del Trabajo, es preocupante la situación laboral que tienen los jóvenes, dado que la mayoría no tiene la oportunidad de obtener un trabajo, siendo así que todo da lugar a una precariedad laboral por la vulnerabilidad en los lugares de trabajo y la informalidad con que los jóvenes acceden a un empleo. Desde un punto de vista general este sector tiene uno de los mayores casos de desempleo en todo el mundo según revela la (ILOSTAT, 2020) siendo personas que no están en ocupación, recibiendo la educación o en capacitación necesaria para la búsqueda de algún puesto de trabajo. 
El desempleo juvenil es uno de los mayores retos que se enfrenta en el marco de su desarrollo social, pues refleja en la concentración de la riqueza, la consolidación de la pobreza y las constantes altas tasas de desempleo, se empieza a vivir una creciente desigualdad social con una precarización del mundo laboral. Según el estudio de la Organización Internacional del Trabajo actualmente en América Latina y el Caribe hay 9,4 millones de jóvenes que están desempleados, 23 millones no están estudiando ni capacitándose, y más de 30 millones sólo consiguen empleo en condiciones precarias.

El estudio de (CEPAL, 2017) determinó que existe un estancamiento en la creación de empleo en varios países de América del Sur (Argentina, Chile, Perú y Uruguay), mientras que en Centroamérica y México esta variable mostró mayor dinamismo. Los sueldos totales del pleno empleo aumentaron en Brasil, Chile, Colombia, Costa Rica, Nicaragua y Uruguay), mientras que disminuyeron en México y Perú. En estos países persistirá el elevado porcentaje de menores de 24 años y la segmentación social con extrema pobreza. Esto significa que en la actualidad hay más de 52 millones de personas entre 15 y 24 años en la fuerza de trabajo regional, incluyendo los ocupados y aquellos que están desocupados, pero buscan activamente un empleo. (OIT, 2020)

El desempleo ha llegado a producir un desequilibrio entre la oferta de mano de obra y la demanda de trabajo. Si en el mercado hay sobreoferta de mano de obra y pocas vacantes para el empleo, se genera un excedente en los recursos humanos (desempleo). Dada la importancia de conocer los problemas o situaciones del Ecuador, es necesario que el gobierno establezca mecanismos para facilitar información estadística confiable que describa en un momento dado la situación de recursos humanos (Vega, 2017).

En Ecuador la falta de experiencia es la principal barrera que enfrentan los jóvenes que buscan un empleo pues en casi cualquier empresa la experiencia es un requisito importante en el que muchos jóvenes carecen. Por eso, se han buscado otras soluciones al desempleo trabajando en negocios familiares, otros trabajan en ramas distintas a la profesión o deciden emprender algo propio, siendo estos sus primeros pasos en el mercado laboral (El Comercio, 2019).

\section{MATERIALES Y MÉTODOS}

Este artículo estudia la condición laboral de personas de entre 15 a 24 años de edad en el territorio nacional. La recolección de información de datos son de fuentes secundarias, correspondientes a la recopilación de evidencias investigativas como la Organización Internacional del Trabajo (OIT), la Comisión Económica para América Latina y el Caribe (CEPAL), el Ministerio de Trabajo, el Instituto Nacional de Estadística y Censos (INEC), entre otras revisiones bibliográficas que a través de un proceso analítico, que permite según el autor (Fernández, 2008) establecer un panorama de las fuentes 
investigadas, tomando como referentes los énfasis propios de formación e investigación del mercado laboral juvenil ecuatoriano y su inclusión económica en el país.

Según (Rivera \& González, 2015) este tipo de fuentes son las que tienen información procesada como fuente primaria, siendo determinada por la interpretación, análisis y extracción y reorganización de la información central. Sin embargo, se plantea la posibilidad de que el concepto de "fuentes secundarias" sea más flexible a medida que se vaya usando dichas fuentes y demostrando su utilidad (Lombardero \& Méndez, 2015).

La metodología de investigación es de carácter mixta, porque se realiza desde dos tipos de enfoques que son el cualitativo y el cuantitativo, interrelacionados en los diferentes puntos a tratar del estudio.

Se utiliza el método descriptivo para detallar sistemáticamente las características de las variables y sus resultados con el fin de generar y perfeccionar categorías conceptuales, comparando los constructos y postulados generados a partir de estos en distintos contextos (Quevedo, 2002).

\section{RESULTADOS Y DISCUSIÓN}

Siendo la inclusión económica esa apertura de oportunidades para el crecimiento económico de los jóvenes es fundamental para lograr una transición al mercado que promueva un sistema inclusivo, tratándose de una asignación eficiente de recursos humanos en lugar de ser solamente una opción de política social.

Para que así se garantice a las personas en riesgo de pobreza y/o exclusión social en la obtención del beneficio de oportunidades de empleo y recursos necesarios para participar plenamente en la vida económica, política, social y cultural, mejorando su nivel de vida y consiguiendo el bienestar que se considere adecuado en la sociedad (MIES, 2019).

A continuación, se observará estos factores que son relevantes para insertar a los jóvenes en el mercado laboral:

\section{Educación}

En la mayoría de los casos, la educación es puesta como una parte fundamental para mejorar la inserción laboral en los jóvenes y se ha constatado que un mayor nivel educativo reduce el riesgo de desempleo. Sin embargo, para algunos también es cuestionable la efectividad de la educación como vehículo que pueda mejorar la inserción laboral (Weller, 2003).

\section{Oferta y demanda laboral}

La disminución de la oferta laboral, o de una demanda competitiva, será un obstáculo porque ésta impide el crecimiento económico y social de la sociedad, y aún peor, cuando a ambos factores se aumenta 
la falta de aplicación de estrategias públicas o privadas que permitan la inclusión de los ciudadanos al mercado de trabajo (Mora \& Zambrano, 2014).

\section{Condiciones socioeconómicas}

El principal motivo de buscar trabajo para un joven es la complementación del presupuesto básico del hogar, lo que es lógico, dado que la gran mayoría no son jefes de hogar. Las personas con escasos recursos y aquellos que no tienen jefe de hogar, son un porcentaje importante de jóvenes cuyo motivo de búsqueda es no solo complementar sino completar el presupuesto básico para subsistir (Weller, 2003).

\section{Instituciones laborales}

Cuando hablamos de instituciones en economía se refiere a leyes, los usos, los hábitos, las costumbres, las normas que rigen las relaciones sociales y económicas entre los agentes económicos. Pueden ser formales o informales. Auto-limitan o restringen el ámbito de actuación de los individuos y los grupos. En definitiva, son las reglas del juego: establecen los comportamientos socialmente aceptables y confieren seguridad a la iniciativa individual y colectiva, debido a que reducen la incertidumbre. Si no hay instituciones no se puede laborar y por tanto no habrá crecimiento en la participación económica.

\section{MERCADO LABORAL EN EL ECUADOR}

\section{Datos estadísticos comportamiento en el mercado laboral en Ecuador}

Tomando la investigación del análisis de la Encuesta Nacional de Empleo, Desempleo y Subempleo ENEMDU, nos presenta los indicadores más importantes que están por el rango de edad en los periodos de marzo 2009 hasta marzo 2019.

PEA en grupos de edad

En el rango de edad, se encontró que la mayor participación en la población económicamente activa desde marzo 2019 fueron los grupos etarios de entre 45 y 64 años y entre 25 y 34 años, con porcentajes de $30.7 \%$ y $25.7 \%$ aproximadamente (BCE, 2019). 
Figura 1. PEA en grupos de edad

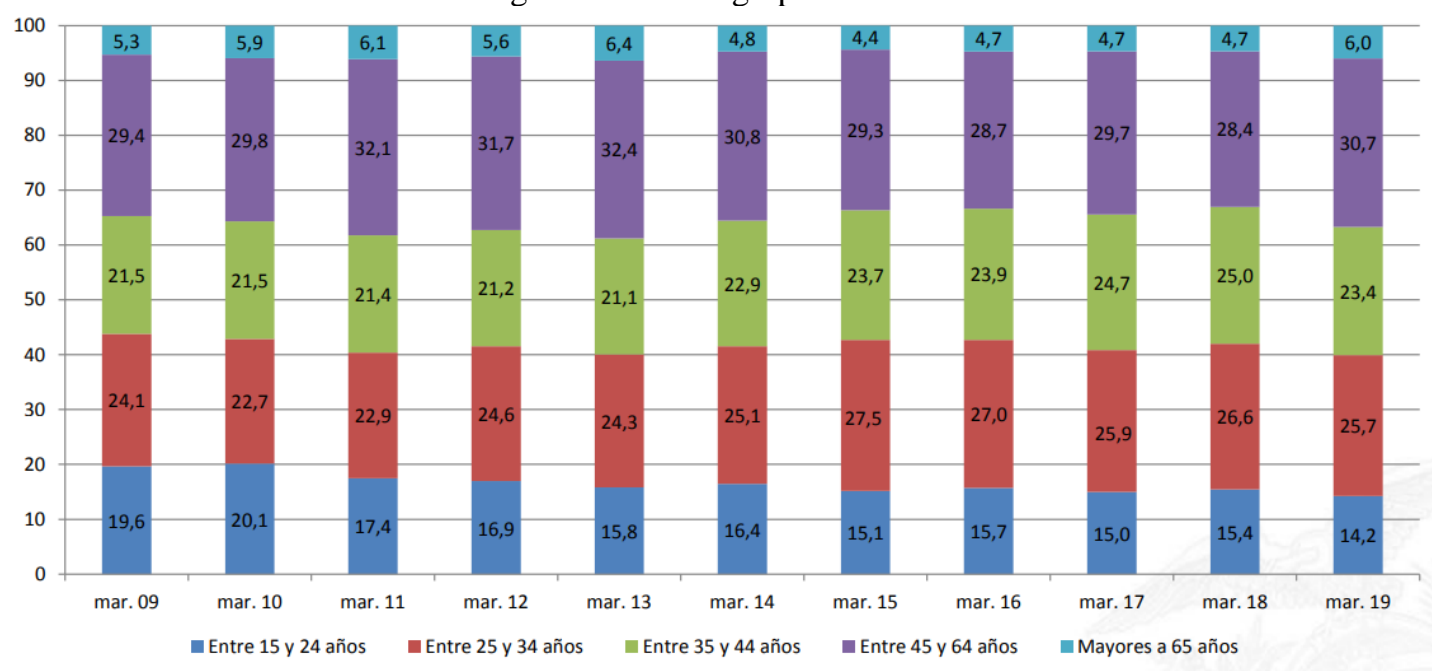

Fuente: INEC y ENEMDU

Elaborado por: Banco Central del Ecuador

Se puede observar en la figura 1 que los jóvenes entre los 15 a 24 años son los que tienen una menor participación en la población económicamente activa, su promedio desde el año 2009 al 2019 es del 18,2\%. La poca interacción de los jóvenes con la PEA sucede probablemente porque la mayoría de ellos están estudiando su bachillerato o ya están en la universidad mientras que según las cifras del Instituto Ecuatoriano de Estadística y Censos, solo 8\% está trabajando y estudiando al mismo tiempo. Por último, están los jóvenes que no estudian ni trabajan siendo un 25,4\% de acuerdo al estudio realizado por la Corporación Andina de Fomento (CAF).

\section{Pleno empleo urbano}

Los grupos de edad con mayor participación en el empleo adecuado/pleno fueron los comprendidos entre los 45 y 64 años, con un porcentaje de $31.6 \%$ y los de entre 25 y 34 años, con un porcentaje de $29.3 \%$. (BCE, 2019)

Figura 2. Tasa de pleno empleo urbano

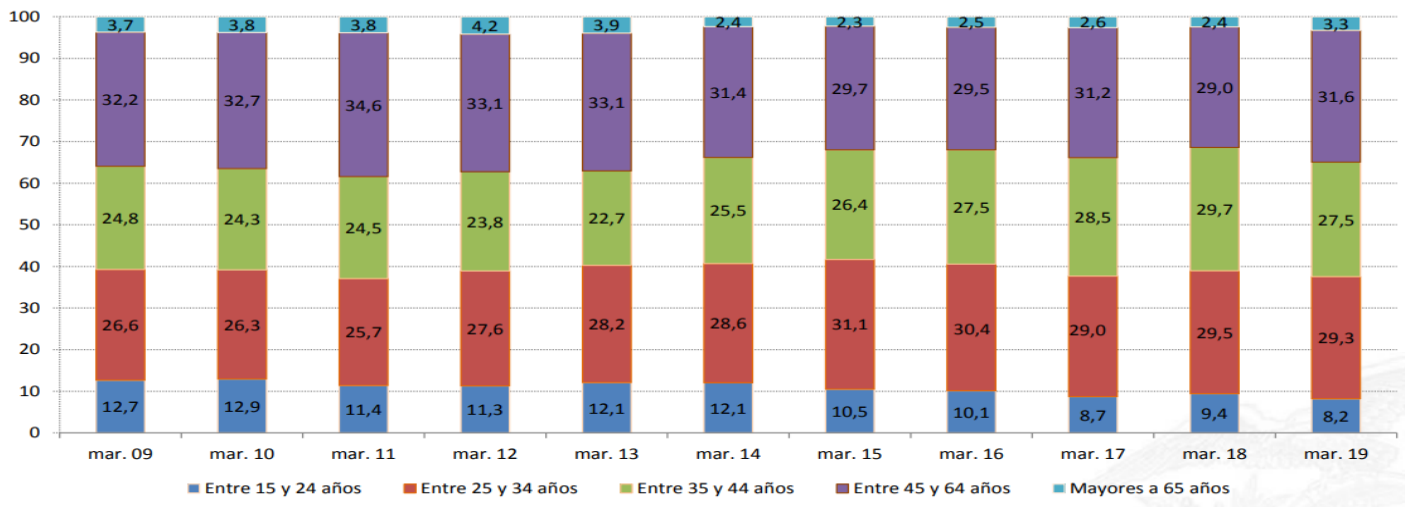

Fuentes: INEC y ENEMDU

Elaborado por: Banco Central del Ecuador 
En la figura 2 se determina que los jóvenes entre los 15 a 24 años poseen su promedio entre desde el año 2009 al 2019 es del 10,1\% de obtener un pleno empleo. Según la encuesta, la mayoría de los jóvenes no posee un empleo formal, esto debido a que es determinante la experiencia laboral que no todos poseen, por lo que las empresas buscan candidatos con conocimientos previos para un mayor desempeño y productividad, en consecuencia, dejando de lado los costos de capacitación si contratan a alguien sin experiencia.

\section{Subempleo urbano}

Los grupos de edad con mayor participación en el subempleo urbano a marzo de 2019 fueron los grupos etarios de entre 45 y 64 años; y, entre 25 y 34 años, con participaciones de $30.3 \%$ y $26.5 \%$, respectivamente. (BCE, 2019)

Figura 3. Tasa de subempleo urbano

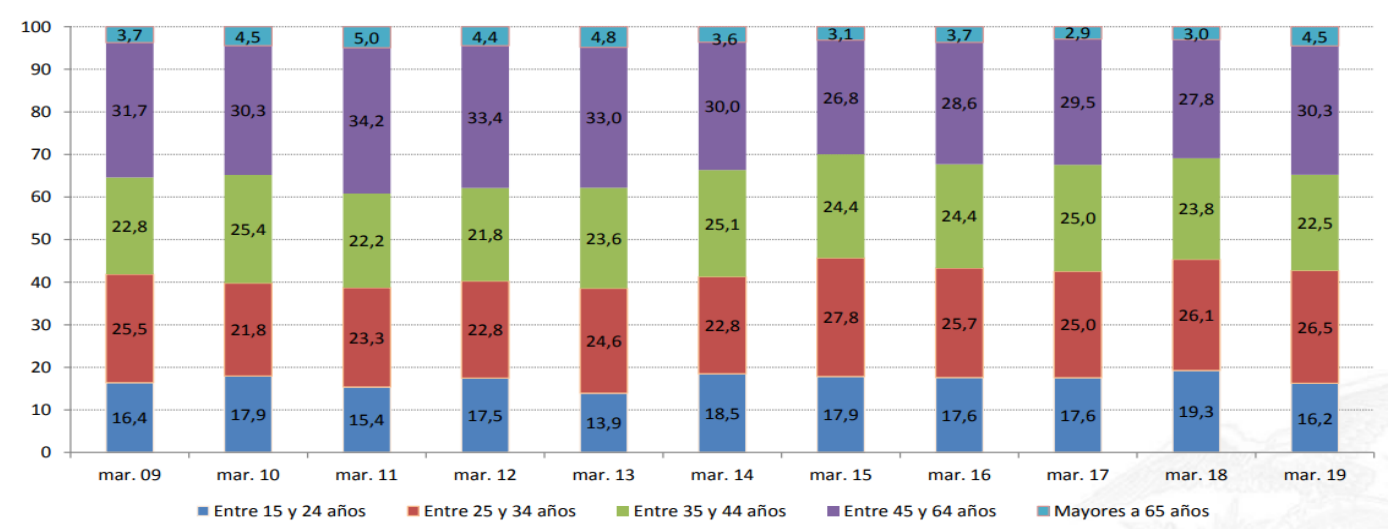

Fuentes: INEC y ENEMDU

Elaborado por: Banco Central del Ecuador

En la figura 3 se observa que los jóvenes entre los 15 a 24 años son los que tienen un menor subempleo urbano, su promedio entre desde el año 2009 al 2019 es de 18,8\%. Estas estimaciones demuestran el análisis del subempleo para los jóvenes ecuatorianos, en su vulnerabilidad a las condiciones laborales precarias, se identifica a mayor nivel de detalle los subconjuntos de jóvenes principalmente en el sector rural (P. Pérez \& M. Hurtado, 2019).

\section{Desempleo nacional}

A marzo de 2019, los grupos de edad con mayor participación en el desempleo fueron los comprendidos entre los 15 y 24 años; y, entre 25 y 34 años con porcentajes de $33.9 \%$ y $32.8 \%$, respectivamente (BCE, 2019). 
Figura 4. Tasa de desempleo nacional

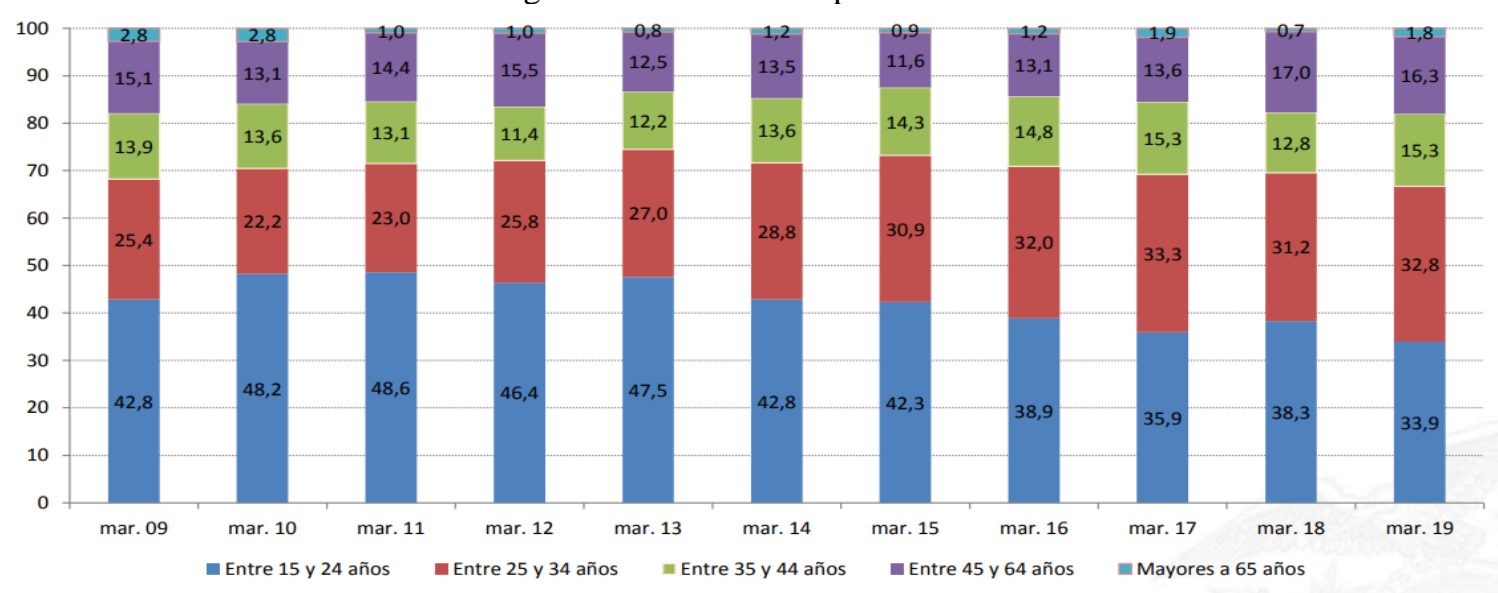

Fuentes: INEC y ENEMDU

Elaborado por: Banco Central del Ecuador

Según la figura 4, los jóvenes entre los 15 a 24 años son los que tienen la mayor cantidad de desempleados en el país, su promedio entre desde el año 2009 al 2019 es de 46,56\%. Dentro de situación laboral en el país, no es la más favorable para los jóvenes, además según una investigación del grupo ADECCO, empresa mundial especializada en recursos humanos, se realizó una encuesta a nivel nacional de las personas que tienen empleo, solo el $27 \%$ de los encuestados posee trabajo fijo, el $25 \%$ es por tiempo indefinido y el $2 \%$ por teletrabajo.

Además, más de $40 \%$ de encuestados aseguró que la poca experiencia fue un factor determinante a la hora de insertarse en el mercado laboral. Por otra parte, $68 \%$ consideró que dentro de la empresa en la que actualmente trabaja no existe posibilidad de crecimiento y desarrollo profesional (Gestión digital, 2019).

Figura 5. Caracterización de la situación laboral de los jóvenes empleados.

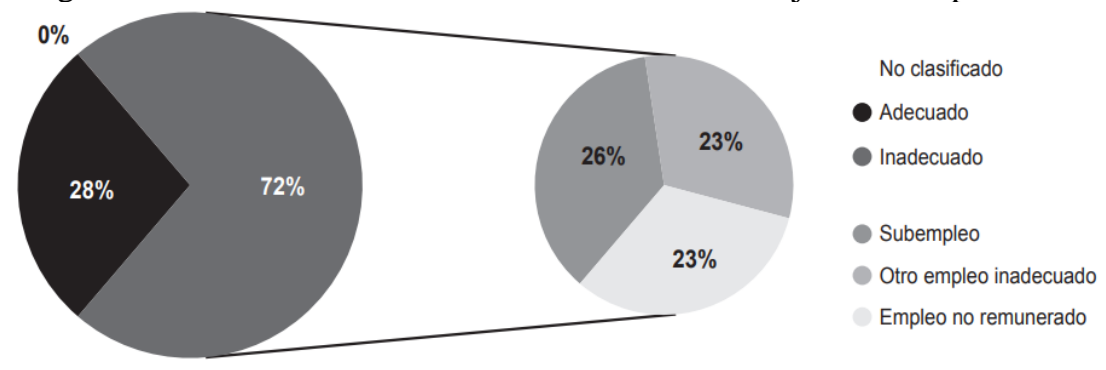

Fuente: INEC Y ENEMDU

Elaborado por: FES-Ecuador

Se puede observar en la figura 5 que sólo $28 \%$ de jóvenes tiene un trabajo pleno o adecuado mientras que el $72 \%$ no ha encontrado trabajo fijo, lo que dificulta las oportunidades de crecimiento profesional y económico (P. Olmedo, 2018). 
El desempleo juvenil tiene impacto a largo plazo sobre el crecimiento económico y la productividad de un país. Desde un punto de vista microeconómico, sufrir episodios de desempleo cuando se es joven, genera, además de una pérdida de ingresos, efectos dañinos permanentes en la carrera de un trabajador (Arrazola et al., 2018).

En cuanto a la evaluación de la Ley Orgánica para la Promoción del Trabajo Juvenil promovida por el Gobierno Nacional en el 2016, Adecco resaltó lo positivo del Estado que ha promovido esta política a favor del impulso del trabajo juvenil por crear el programa "Mi Primer Empleo" y el proyecto para incentivar el empleo juvenil en el sector privado "Empleo Joven", se tuvo un impulso exitoso constituyéndose como la mayor red de datos de este grupo, pero uno de sus limitantes fue no sumar con los filtros principales robustos que aseguran la identidad de las características específicas solicitadas por las empresas y esto ocasionó que el mercado reprima la oportunidad de obtener puestos de empleo y recurra a sus propios esquemas de incorporación, dando prioridad a perfiles con experiencia previa, independientemente de la edad con la que contarán (ADECCO \& Lima, 2018).

\section{CONCLUSIONES}

En el Ecuador, aunque han creado varios programas como "Mi Primer Empleo" y "Empleo Joven" que buscan potenciar el mercado laboral juvenil, no todo dependerá de los empleados sino de los empleadores en su mayoría, estableciendo requisitos con los cuales muchos jóvenes no cuentan, por la falta de experiencia, títulos de tercer y cuarto grado, disponibilidades económicas y sociales, entre otros.

Como consecuencia, ha disminuido el pleno empleo, el subempleo por lo contrario el desempleo ha aumentado; y esto no es alentador, por lo tanto se necesita mayor desarrollo de propuestas del Estado para mitigar el desempleo juvenil, ya sea con capacitaciones gratuitas o bajo costo a nivel nacional (mayormente en sitios vulnerables) porque un mejor nivel de formación ofrece mejoras no solo en la productividad, sino también en la calidad de vida de los trabajadores jóvenes que acceden a su primer empleo, teniendo así un mejor crecimiento laboral, creando convenios con las empresas públicas y privadas, regulando las posibles precariedades laborales en que pueden existir en el entorno de trabajo. 


\section{REFERENCIAS}

ADECCO, \& Lima, S. (2018). TERMÓMETRO DE LA SITUACIÓN LABORAL DE LOS JÓVENES EN ECUADOR. LinkenIn. Published. https://www.linkedin.com/pulse/term\%C3\%B3metro-de-lasituaci\%C3\%B3n-laboral-los-j\%C3\%B3venes-en-ecuador-lima/?originalSubdomain=es

ARRAZOLA, M., GALÁN, S., HEVIA, J., \& Universidad Rey Juan Carlos. (2018). Desempleo juvenil en España situación, consecuencias e impacto sobre la vida laboral de los adultos. Dialnet, 156, 62-75. https://dialnet.unirioja.es/servlet/articulo?codigo=6518997

BCE. (marzo de 2019). REPORTE TRIMESTRAL DE MERCADO LABORAL. Obtenido de https://contenido.bce.fin.ec/documentos/Estadisticas/SectorReal/Previsiones/IndCoyuntura/Empleo/imle 201901.pdf

CEPAL. (1998, julio). EMANCIPACIÓN JUVENIL: TRAYECTORIAS y DESTINOS. Oficina de Montevideo.https://repositorio.cepal.org/bitstream/handle/11362/28642/LCmvdR154rev1_es.pdf?seque nce $=1$

CEPAL / OIT. (2017, octubre). La transición de los jóvenes de la escuela al mercado laboral (Número 17). Coyuntura Laboral en América Latina y el Caribe. https://repositorio.cepal.org/bitstream/handle/11362/42250/1/S1700893_es.pdf

EL COMERCIO. (2019, 21 julio). Los jóvenes entre 15 y 24 años de edad son los que más dificultad tienen de conseguir empleo. https://www.elcomercio.com/actualidad/jovenes-oportunidades-laboralesquito.html

Ecuador: El 25,4\% de jóvenes de 18 a 24 años son ninis, ni estudian ni trabajan. (2017, 8 enero). El Universo. https://www.eluniverso.com/noticias/2017/01/08/nota/5984536/254-jovenes-18-24-anos-sonninis-ni-estudian-ni-trabajan/

Fernández, M. (2008). El proceso investigativo en el estado del arte de la Maestría en Educación de la Universidad Santo Tomás. Fundación Dialnet. https://dialnet.unirioja.es/servlet/articulo?codigo $=4037187$

Gestión digital. (2019). El panorama laboral aún es complejo para los jóvenes en Ecuador. Revista Gestión, Multiplica. https://www.revistagestion.ec/economia-y-finanzas-analisis/el-panorama-laboralaun-es-complejo-para-los-jovenes-en-ecuador

ILOSTAT. (noviembre, 2020). Estadísticas sobre el trabajo juvenil. ILO. https://ilostat.ilo.org/es/topics/youth/

Kiziryan, M. (2018). Obtenido de Mercado Laboral: https://economipedia.com/definiciones/mercadolaboral.html

Lombardero, P., \& Méndez, M. (2015). LAS FUENTES SECUNDARIAS PARA EL HISTORIADOR: UNA REFLEXIÓN A PARTIR DE LO DIGITAL Y LO LITERARIO. Tiempo y Sociedad, 21, 159-176. https://es.slideshare.net/pablofolgueira3/fuentes-secundarias-para-el-historiador-una-reflexin-a-partirde-lo-digital-y-lo-literario-2015 
Mora, J., \& Zambrano, J. (2014). Inserción al mercado laboral que contribuye al crecimiento económico del Cantón Babahoyo. Published. https://dspace.uniandes.edu.ec/handle/123456789/2469

OIT. (2020, marzo). Desempleo, informalidad e inactividad asedian a los jóvenes en América Latina y el Caribe. Informe Mundial sobre el Empleo Juvenil 2020. https://www.ilo.org/americas/sala-deprensa/WCMS_738631/lang--es/index.htm

Olmedo, P. (2018, mayo). El empleo en el Ecuador - Una mirada a la situación y perspectivas para el mercado laboral actual (N.o 14525). Friedrich Ebert Stiftung Ecuador FES. https://library.fes.de/pdffiles/bueros/quito/14525.pdf

Petter Pérez, L. \& Moreno Hurtado, C. (2019). Subempleo en el mercado laboral juvenil en Ecuador. $\begin{array}{llll}\text { Revista } & \text { nuestrAmérica, } & \text { 265-280. }\end{array}$ https://www.redalyc.org/jatsRepo/5519/551957774014/551957774014.pdf

Quecedo, R. (2002). Introducción a la metodología de investigación cualitativa. Redalyc.org. https://www.redalyc.org/articulo.oa?id=17501402

Rivera, M., \& González, M. (2015). Fuentes de Información. Universidad Autónoma del Estado de Hidalgo.

Published.

https://repository.uaeh.edu.mx/bitstream/bitstream/handle/123456789/16700/LECT132.pdf

Vega, A. C. (octubre, 2017). Análisis de la política gubernamental de apoyo al sector empresarial en la reducción del desempleo. En el caso del cantón Machala en el periodo 2011 - 2015. Universidad Católica de Santiago de Guayaquil. http://repositorio.ucsg.edu.ec/handle/3317/9578

Weller, J. (diciembre, 2003). La problemática inserción laboral de los y las jóvenes. Mayol Ediciones S.A. https://repositorio.cepal.org/bitstream/handle/11362/5391/S0312870_es.pdf?sequence=1\&isAllowed=y 